\title{
In Vitro Comparison of Diagnostic Accuracy of DIAGNOdent and Digital Radiography for Detection of Secondary Proximal Caries Adjacent to Composite Restorations
}

\author{
Zahra Ghoncheh ${ }^{1}$, Zahra Zonouzy ${ }^{2}$, Nazanin Kiomarsi ${ }^{3}$, Mohammad Javad Kharazifard ${ }^{2}$, Nasim Chiniforush ${ }^{4 *}$ \\ ${ }^{1}$ Department of Oral and Maxillofacial Radiology, Dental faculty, Tehran University of Medical Sciences, International \\ Campus, Tehran, Iran \\ ${ }^{2}$ Tehran University of Medical Sciences, International Campus, Tehran, Iran \\ ${ }^{3}$ Department of Operative Dentistry, Dental Faculty, Tehran University of Medical Sciences, International Campus, Tehran, \\ Iran \\ ${ }^{4}$ Laser Research Center of Dentistry (LRCD), Dental Research Institute, Tehran University of Medical Sciences, International \\ Campus, Tehran, Iran
}

\author{
*Correspondence to \\ Nasim Chiniforush, \\ DDS, PhD of Laser Dentistry; \\ Laser Research Center of Dentistry, \\ Dentistry Research Institute, Tehran \\ University of Medical Sciences, \\ Tehran, Iran. \\ Tel/ Fax: +98 2188994824; \\ Email: \\ n-chiniforush@farabi.tums.ac.ir
}

Published online 27 September 2017

\begin{abstract}
Introduction: Early detection of secondary proximal caries is critical for the preservation of tooth vitality. This study sought to assess and compare the diagnostic accuracy of DIAGNOdent and digital radiography for detection of secondary proximal caries adjacent to composite restorations. Methods: Sixty extracted molars including 30 teeth with carious lesions and 30 sound teeth were randomly selected. Class II cavities were prepared in all teeth and carious dentin was intentionally left in the gingival floor of cavities in 30 carious teeth. All cavities were restored with composite resin. The teeth were mounted in wax blocks (three teeth per block) and examined for caries using (DIAGNOdent kaVo Dental, Biberach, Germany). Digital radiographs using DIGORA photostimulable phosphor (PSP) plates (Soredex Corporation, Helsinki, Finland) were obtained from all teeth using the parallel technique and were evaluated by 4 observers. Repeated measure analysis of variance (ANOVA) was applied to calculate sensitivity and specificity values of the two diagnostic techniques. Receiver operating characteristic (ROC) curve was plotted for DIAGNOdent results and based on that, the cutoff points were determined.

Results: The sensitivity and specificity values at the cut-off point of 10.5 were $0.622 \pm 0.038$ and $0.822 \pm 0.077$ for DIAGNOdent and $0.591 \pm 0.093$ and $0.891 \pm 0.083$ for digital radiography, respectively. The area under the ROC curve was 0.7 for DIAGNOdent. Weighted kappa revealed moderate to almost perfect intra-observer agreement (0.46-0.99). Intraclass correlation coefficient (ICC) for DIAGNOdent was calculated to be 0.88 .

Conclusion: No statistically significant difference was noted in diagnostic accuracy of DIAGNOdent and digital radiography for detection of secondary proximal caries adjacent to composite restorations. Thus, DIAGNOdent may be used as an adjunct diagnostic tool for detection of secondary proximal caries beneath composite restorations.

Keywords: Diagnostic accuracy; Proximal caries; DIAGNOdent; Digital radiography; Composite restoration.
\end{abstract}

\section{Introduction}

Despite the recent advances in preventive dentistry and improved quality of restorative materials, clinicians still witness a high incidence of secondary caries. Secondary carious lesions account for $40 \%-70 \%$ of restoration exchanges. ${ }^{1}$ According to the FDI World DentalFederation, secondary caries is defined as "positively diagnosed lesion at the margins of an existing restoration."2 Areas prone to plaque accumulation such as the margins of restorations are most susceptible to development of secondary caries. ${ }^{3}$ Secondary caries refers to a thin carious lesion in the enamel or dentin at the cavity floor-restoration interface. ${ }^{4}$ Clinicians often detect secondary caries via observation, probing with a dental explorer and radiography. Marginal discoloration of restorations is not a reliable indicator of secondary caries. Examination and probing of restoration margins with an explorer tip must be done with caution and utmost care must be taken not to damage the tooth

Please cite this article as follows: Ghoncheh Z, Zonouzy Z, Kiomarsi N, Kharazifard MJ, Chiniforush N. In vitro comparison of diagnostic accuracy of DIAGNOdent and digital radiography for detection of secondary proximal caries adjacent to composite restorations. I Lasers Med Sci. 2017;8(4):172-176. doi:10.15171/jlms.2017.31. 
structure or the restorative material. Moreover, it should be noted that a sharp explorer tip may be caught in almost any fissure or groove and therefore, it does not necessarily mean presence of caries. ${ }^{4}$ Bitewing radiography is the most commonly used diagnostic technique for detection of secondary caries. ${ }^{5}$ However, the effect of the level of education and experience of the observer on accurate interpretation of radiographs and also the confounding effect of artifacts of restorative materials are all among the limitations of radiography. ${ }^{6}$ In the clinical setting, secondary caries often remain undetected until they form a cavity or result in loss of a significant portion of tooth structure. Thus, new diagnostic methods have been introduced for early detection of caries including electrical conductance measurement, laser florescence (LF), digital radiography and enhancement of digital radiographs. ${ }^{1-7}$ DIAGNOdent (LF pen) is a diagnostic tool for caries detection operating based on LF. It quantifies the extent of carious lesions and provides numerical data. The tooth surface is irradiated with red light (at $655 \mathrm{~nm}$ wavelength) generated by a diode laser and transferred to the tooth via an optical fiber. Laser light is absorbed by the tooth surface and is then reflected due to the fluorescence characteristic of the tooth structure. The intensity of fluorescence reflected from the carious areas is higher than that of sound tooth structure. The fluorescence collected from bacterial metabolites in the carious lesion such as the porphyrins has a wavelength close to that of red light (wavelength of $655 \mathrm{~nm}$ ) and thus, a higher number is displayed on the digital display monitor of DIAGNOdent. The deeper the carious lesion, the higher the number displayed. ${ }^{8}$

Previous studies have mostly focused on the application of DIAGNOdent for detection of primary caries and the efficacy of this diagnostic tool has been confirmed in many studies ${ }^{9,10}$ and rejected by some other studies. ${ }^{11,12}$ Studies on the application of DIAGNOdent for detection of secondary caries beneath composite restorations are limited and there is a gap of information in this regard in dental literature. Boston was the first to evaluate secondary caries beneath composite restorations; although he evaluated secondary caries in the occlusal surface. ${ }^{13}$ Later on, some other researchers mainly focused on detection of occlusal secondary caries and caries around amalgam restoration margins. ${ }^{14-16}$

Considering the importance of detecting secondary caries adjacent to composite restorations in the proximal areas (particularly in the gingival floor), the current study focused on this particular area. To the best of our knowledge, similar previous studies focusing on this topic are scarce. Rodrigues et $\mathrm{al}^{17}$ were the only researchers who compared the diagnostic efficacy of DIAGNOdent and radiography for detection of secondary caries around composite restorations. They recommended the use of DIAGNOdent as an adjunct diagnostic tool for detection of secondary caries. The difference between their study and ours was that they used conventional film-based radiography.

Since, currently, digital sensors are used for image capture and no comparison has been made between the efficacy of DIAGNOdent and digital radiography for detection of secondary proximal caries, the current in vitro study was undertaken to assess and compare the diagnostic accuracy of DIAGNOdent and digital radiography with photostimulable phosphor (PSP) plates (DIGORA) in detection of secondary caries adjacent to composite restorations.

\section{Methods}

Preparation of Teeth

Sixty extracted human molar teeth, stored in saline solution, were used in this study. Thirty teeth with proximal caries and 30 sound teeth were selected. Dental calculus was precisely removed using a scaler and the teeth were rinsed under running water for 15 seconds. Classic class II cavities were prepared in all teeth. Carious dentin was intentionally left in the gingival floor of cavities in carious teeth ( $2 \mathrm{~mm}$ depth). The 30 sound teeth were checked using a dental explorer to ensure absence of caries. The cavities were prepared using 008 straight diamond bur and high-speed hand piece along with water spray. The cavities were restored with composite resin without etching and bonding. The teeth were randomly coded from 1 to 60 and mounted in wax blocks. Three teeth were mounted in each block simulating proximal contacts. A total of 60 proximal surfaces were evaluated.

\section{Obtaining Radiographs}

All radiographs were obtained using DIGORA ${ }^{\circledR}$ Optime (Soredex Corporation) and size 2 PSP plates measuring 30 x $40 \mathrm{~mm}$. The $\mathrm{x}$-ray dental machine MINRAY ${ }^{\circledR}$ (Soredex, Helsinki, Finland) was adjusted to exposure settings of 70 $\mathrm{kVp}, 1 \mathrm{~mA}$ and 0.1 second radiation time. Radiographs were captured in faciolingual direction using the parallel technique. After exposure, PSP plates were scanned using Soredex Digora ${ }^{\circledR}$ Optime scanner with standard resolution. The distances from the $\mathrm{x}$-ray tube to the object and from the sensor plate to the object were $45 \mathrm{~cm}$ and $1 \mathrm{~cm}$, respectively. To simulate the absorption properties of soft tissue, a $4 \mathrm{~cm}$-thick acrylic block (polymethyl methacrylate) measuring $15 \times 15 \mathrm{~cm}$ was placed between the $\mathrm{x}$-ray tube and dental blocks. ${ }^{18}$

\section{Interpretation of Radiographs}

All digital radiographs were displayed on a 19-inch (Samsung, SyncMaster) monitor with a resolution of 1360 $\times 768$ pixels using SCANORA Lite software. Radiographs were evaluated in a semi-dark room by four observers: an oral and maxillofacial radiologist, a restorative dentist, a general dentist with 20 years of clinical experience and a senior dental student. The observers were informed about the methodology and objectives of the study. They were 
allowed to adjust the density and contrast of images and no time limitation was set for the observation of images. Observers evaluated the images in terms of presence or absence of caries in the gingival floor of the restorations. To assess the intra-observer reproducibility, the same images were evaluated by the observers again after a onemonth interval under similar conditions.

\section{DIAGNOdent Measurement}

Prior to using DIAGNOdent, the teeth were cleaned using an air polisher and prophylactic paste for 10 seconds and then rinsed under running water. The proximal surfaces were examined by a wedge-shaped sapphire fiber tip (thickness of $0.4 \mathrm{~mm}$ and width of $1.1 \mathrm{~mm}$ ). DIAGNOdent was calibrated prior to measurements according to the manufacturer's instructions. The tip of the device was moved below the contact point from the buccal towards the lingual surface. This was repeated from the lingual towards the buccal as well and the highest value was recorded. Measurements were repeated 3 times for each tooth.

\section{Statistical Analysis}

Sensitivity and specificity parameters were calculated to assess and compare the diagnostic efficacy of DIAGNOdent and digital radiography for detection of secondary caries. The area under the receiver operating characteristic (ROC) curve was calculated for DIAGNOdent data. Repeated measure analysis of variance (ANOVA) was applied to compare the sensitivity and specificity of the two diagnostic methods. $P<0.05$ was considered statistically significant. Weighted kappa was calculated to determine intra and inter-observer agreement. Intraclass correlation coefficient (ICC) was used to evaluate the reproducibility of DIAGNOdent (two-way random, absolute agreement).

\section{Results}

The sensitivity and specificity of DIAGNOdent and digital radiography for detection of secondary caries were calculated and the cutoff points were determined (Table 1). The cutoff points in ROC curve for DIAGNOdent were 8.5, 9.5 and 10.5; which were considered as diagnostic cutoff points. Based on Table 1, although the sensitivity at the cutoff point of 8.5 for DIAGNOdent was significantly higher than that for digital radiography $(0.73 \pm 0.001$ vs $0.59 \pm 0.09 ; P=.01)$, the specificity at this cutoff point for DIAGNOdent was significantly lower than that for digital radiography $(0.57 \pm 0.03$ vs. $0.89 \pm 0.08 ; P=0)$. In terms of sensitivity, no significant difference existed at the cutoff point of 9.5 between DIAGNOdent and digital radiography ( $0.68 \pm 0.3$ vs. $0.59 \pm 0.09 ; P=0.06)$, but the specificity of DIAGNOdent at this cutoff point was lower than that of digital radiography $(0.67 \pm 0.03$ vs $0.89 \pm$ $0.08 ; P=0.002)$. The area under the ROC curve was found to be 0.7 for DIAGNOdent (Figure 1). Intraobserver reproducibility for observers 1 to 4 was $0.88,0.46,0.73$ and 0.99 , respectively for digital radiography. The mean interobserver reproducibility was calculated to be 0.73 for digital radiography. ICC for DIAGNOdent was reported to be 0.88 .

\section{Discussion}

Diagnosis of secondary caries is a challenging topic due to the increasing use of restorative materials. Early detection of secondary caries is critical for a prompt preventive measure or making a decision to exchange the restoration. ${ }^{4,13}$ The current study assessed and compared the diagnostic efficacy of digital radiography and DIAGNOdent for detection of secondary caries beneath composite restorations. Our results confirmed the optimal efficacy of DIAGNOdent for detection of secondary proximal caries adjacent to composite restorations. However, its diagnostic efficacy was not significantly different from that of digital radiography; this finding is in line with the results of some other studies. ${ }^{6,18,19}$

Bonding agents, applied to the cavity floor beneath composite restorations may compromise accurate radiographic detection of secondary caries and even result in misdiagnosis, especially if placed in a thick layer. Thus, in the current study, similar to that of Kositbowornchai et al, these materials were not used. ${ }^{6,20}$

Sensitivity and specificity of a diagnostic technique are mainly calculated with a cutoff point. Thus, for accurate determination of the cut-off point, three methods were used in the current study. The first method was to find a

Table 1. Sensitivity and Specificity for Digital Radiography (by the 4 Observers) and DIAGNOdent Using Different Cut-off Points

\begin{tabular}{lll}
\hline & Sensitivity & Specificity \\
\hline Radiographic examination & $0.59 \pm 0.09$ & $0.89 \pm 0.08$ \\
DIAGNOdent 8.5 & $0.73 \pm 0.001$ & $0.57 \pm 0.03$ \\
DIAGNOdent 9.5 & $0.68 \pm 0.03$ & $0.67 \pm 0.03$ \\
DIAGNOdent 10.5 & $0.62 \pm 0.03$ & $0.82 \pm 0.07$ \\
\hline
\end{tabular}

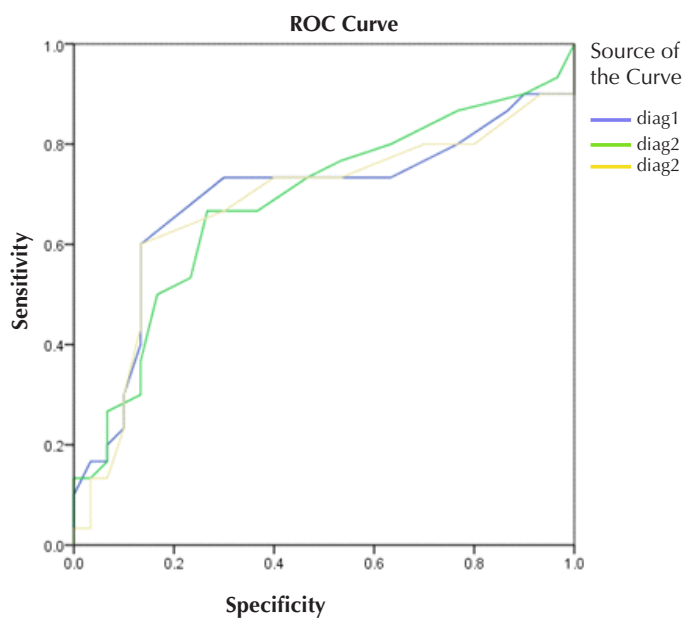

Figure 1. Roc Curve for DIAGNOdent on Secondary Caries Detection (in 3 Times Measurements). 
cutoff point in the ROC curve. Using this curve, 2 points with significant properties were found: point 8.5 with a sensitivity higher and a specificity lower than those of digital radiography and point 10.5 with no statistically significant difference with digital radiography in this regard. The second method was detection of a point where specificity yielded one. The specificity at point 29 was found to be 1 in all three measurements. Based on this specificity value, the point 29 was the most suitable point for presence of secondary caries. In a study by Rodrigues et al, the point 30 was determined to be the most suitable point in terms of specificity. In our study, the sensitivity and specificity of DIAGNOdent at the cut-off point of 10.5 were similar to those of digital radiography. Some previous in-vitro studies have also reported almost equal sensitivity and specificity values for DIAGNOdent and digital radiography. ${ }^{21}$

The area under the ROC curve, indicating the correlation of sensitivity and specificity, was calculated to be 0.71 for DIAGNOdent. The possible range for this value (area under the ROC curve) is $0.5-1 .^{22}$ Thus, the value obtained in our study is moderate and close to the value reported by Rodrigues et al. ${ }^{17}$

The kappa statistics showed excellent intra-observer agreement for observers 1 and 4, and observers 2 and 3 had moderate and substantial intra-observer agreements, respectively. Also, the mean inter-observer kappa coefficient was found to be 0.73 ; which is a moderate value. $^{23}$

DIAGNOdent was expected to have a reproducibility coefficient close to 1 . In the current study, ICC was found to be 0.88 ; which indicates high reproducibility of this tool. This finding is close to the results of Rodrigues, studying secondary proximal caries and Lussi et al evaluating primary proximal caries. ${ }^{17,24}$

The results of this study indicated higher reproducibility of DIAGNOdent compared to digital radiography. This finding indicates that the clinical experience and knowledge of observers may affect the interpretation of digital radiographs; whereas, based on studies by Kuhnisch et $\mathrm{al}^{25}$ and Hamishaki et $\mathrm{al},{ }^{5}$ the results of DIAGNOdent are not influenced by the operator's skills. Some studies have demonstrated that DIAGNOdent (in comparison to radiography) enhances the detection of secondary caries around amalgam restorations. ${ }^{14,16}$ However, some others do not recommend the use of DIAGNOdent for detection of residual caries. ${ }^{26}$ Krause et al demonstrated that DIAGNOdent had limitations for detection of residual caries close to dental pulp ${ }^{27}$; this indicates that in the clinical setting, many factors may affect the performance of DIAGNOdent.

Some studies have reported that the wedge-shaped tip design (B tip) of DIAGNOdent used in the current study increases the sensitivity for detection of caries. On the other hand, the contact point simulation in vitro does not exactly resemble the actual contact points in vivo. Moreover, the access of DIAGNOdent (B tip) to contact areas in vitro is much easier than in vivo. Furthermore, probing the proximal surfaces of Class II restorations with DIAGNOdent still remains a challenge. It must be noted that prior to examining the teeth surfaces with DIAGNOdent, the teeth and restoration surfaces should be thoroughly polished because calculus, deposits and stain may lead to false positive results. ${ }^{27,28}$

PSP plates (Digora system) were used for image capture in the current study, which is in contrast to the methodology of previous studies; however, our obtained results confirmed those of previous investigations. ${ }^{6}$ In order to enhance the detection of caries and decrease the number of bitewing radiographs required, DIAGNOdent is recommended to be used in conjunction with bitewing radiography.

DIAGNOdent at a cut-off point of 8.5 , with a higher sensitivity and a lower specificity than that of digital radiography, can be used as the first line diagnostic method for field screening tests when a high number of samples should be screened in a short period of time. Since there is a high risk of false positive results, DIAGNOdent should be preferably used in conjunction with other diagnostic techniques.

\section{Conclusion}

DIAGNOdent and digital radiography have equal diagnostic efficacy for detection of secondary proximal caries adjacent to composite restorations. Future studies are required to further scrutinize the diagnostic efficacy of the combination of these two methods for detection of secondary caries.

\section{Ethical Considerations}

This study has been approved by ethical committee of Tehran University of Medical Sciences, International Campus.

\section{Conflict of Interests}

None.

\section{References}

1. Bamzahim M, Shi XQ, Angmar-Mansson B. Secondary caries detection by DIAGNOdent and radiography: a comparative in vitro study. Acta Odontol Scand. 2004;62(1):61-64.

2. The Federation Dentaire Internationale (Special Commission on Oral and Dental Statistics). General principles concerning the international standardization of dental caries statistics. Int Dent J. 1962;12:65.

3. Lima FG, Romano AR, Correa MB, Demarco FF. Influence of microleakage, surface roughness and biofilm control on secondary caries formation around composite resin restorations: an in situ evaluation. J Appl Oral Sci. 2009;17(1):61-65. 
4. Bamzahim M, Aljehani A, Shi XQ. Clinical performance of DIAGnodent in the detection of secondary carious lesions. Acta Odontol Scand. 2005;63(1):26-30.

5. Hamishaki KS, Chiniforush N, Monzavi A, Khazarazifard MJ. An in vivo comparison of two diagnostic methods in secondary caries detection. J Dent (Tehran). 2014;11(1):1721.

6. Kositbowornchai S, Sukanya C, Tidarat T, Chanoggarn T. Caries detection under composite restorations by laser fluorescence and digital radiography. Clin Oral Investig. 2013;17(9):2079-2084. doi:10.1007/s00784-012-0908-9.

7. Pontual AA, de Melo DP, de Almeida SM, Boscolo FN, Haiter Neto F. Comparison of digital systems and conventional dental film for the detection of approximal enamel caries. Dentomaxillofac Radiol. 2010;39(7):431436. doi:10.1259/dmfr/94985823.

8. Lussi A, Hibst R, Paulus R. DIAGNOdent: an optical method for caries detection. J Dent Res. 2004;83 Spec No C:C80-83.

9. Sridhar N, Tandon S, Rao N. A comparative evaluation of DIAGNOdent with visual and radiography for detection of occlusal caries: an in vitro study. Indian J Dent Res. 2009;20(3):326-331. doi:10.4103/0970-9290.57376.

10. Toraman Alkurt M, Peker I, Deniz Arisu H, Bala O, Altunkaynak B. In vivo comparison of laser fluorescence measurements with conventional methods for occlusal caries detection. Lasers Med Sci. 2008;23(3):307-312. doi:10.1007/s10103-007-0486-2.

11. Angnes G, Angnes V, Grande RH, Battistella M, Loguercio $\mathrm{AD}$, Reis A. Occlusal caries diagnosis in permanent teeth: an in vitro study. Braz Oral Res. 2005;19(4):243-248. doi:/ S1806-83242005000400002.

12. Fung L, Smales R, Ngo H, Moun G. Diagnostic comparison of three groups of examiners using visual and laser fluorescence methods to detect occlusal caries in vitro. Aust Dent J. 2004;49(2):67-71.

13. Boston DW. Initial in vitro evaluation of DIAGNOdent for detecting secondary carious lesions associated with resin composite restorations. Quintessence Int. 2003;34(2):109116.

14. Neuhaus KW, Rodrigues JA, Seemann R, Lussi A. Detection of proximal secondary caries at cervical class II-amalgam restoration margins in vitro. J Dent. 2012;40(6):493-499. doi:10.1016/j.jdent.2012.02.014.

15. Chu CH, Lo EC, You DS. Clinical diagnosis of fissure caries with conventional and laser-induced fluorescence techniques. Lasers Med Sci. 2010;25(3):355-362. doi:10.1007/s10103-009-0655-6.

16. Ando M, Gonzalez-Cabezas C, Isaacs RL, Eckert GJ, Stookey GK. Evaluation of several techniques for the detection of secondary caries adjacent to amalgam restorations. Caries Res. 2004;38(4):350-356. doi:10.1159/000078181.

17. Rodrigues JA, Neuhaus KW, Hug I, Stich H, Seemann R, Lussi A. In vitro detection of secondary caries associated with composite restorations on approximal surfaces using laser fluorescence. Oper Dent. 2010;35(5):564-571. doi:10.2341/09-332-1.

18. Caldas Mde P, Ramos-Perez FM, de Almeida SM, HaiterNeto F. Comparative evaluation among different materials to replace soft tissue in oral radiology studies. J Appl Oral Sci. 2010;18(3):264-267.

19. Lennon AM, Buchalla W, Switalski L, Stookey GK. Residual caries detection using visible fluorescence. Caries Res. 2002;36(5):315-319.

20. Kursun S, Dinc G, Oztas B, Yuksel S, Kamburoglu K. The visibility of secondary caries under bonding agents with two different imaging modalities. Dent Mater J. 2012;31(6):975-979.

21. Kuhnisch J, Berger S, Goddon I, Senkel H, Pitts N, HeinrichWeltzien R. Occlusal caries detection in permanent molars according to WHO basic methods, ICDAS II and laser fluorescence measurements. Community Dent Oral Epidemiol. 2008;36(6):475-484. doi:10.1111/j.16000528.2008.00436.x.

22. HanleyJA, McNeil BJ. The meaning and use of the area under a receiver operating characteristic (ROC) curve. Radiology. 1982;143(1):29-36. doi:10.1148/radiology.143.1.7063747.

23. Landis JR, Koch GG. The measurement of observer agreement for categorical data. Biometrics. 1977;33(1):159174.

24. Lussi A, Hack A, Hug I, Heckenberger H, Megert B, Stich H. Detection of approximal caries with a new laser fluorescence device. Caries Res. 2006;40(2):97-103. doi:10.1159/000091054.

25. Kuhnisch J, Ziehe A, Brandstadt A, Heinrich-Weltzien R. An in vitro study of the reliability of DIAGNOdent measurements. J Oral Rehabil. 2004;31(9):895-899. doi:10.1111/j.1365-2842.2004.01315.x.

26. Yazici AR, Baseren M, Gokalp S. The in vitro performance of laser fluorescence and caries-detector dye for detecting residual carious dentin during tooth preparation. Quintessence Int. 2005;36(6):417-422.

27. Krause F, Braun A, Eberhard J, Jepsen S. Laser fluorescence measurements compared to electrical resistance of residual dentine in excavated cavities in vivo. Caries Res. 2007;41(2):135-140. doi:10.1159/000098047.

28. Rodrigues JA, Hug I, Diniz MB, Lussi A. Performance of fluorescence methods, radiographic examination and ICDAS II on occlusal surfaces in vitro. Caries Res. 2008;42(4):297-304. doi:10.1159/000148162. 